\title{
Blastomycosis-like pyoderma in an immunocompetent patient
}

\author{
Fatma Sahli ${ }^{1}$, Noureddine Litaiem ${ }^{2}$, Soumaya Gara ${ }^{2}$, Olfa Charfi ${ }^{2}$, soumaya Rammeh ${ }^{2}$, and \\ Faten Zeglaoui ${ }^{2}$ \\ ${ }^{1}$ Hôpital Charles Nicolle \\ ${ }^{2}$ Charles Nicolle Hospital
}

May 28, 2021

\begin{abstract}
Bastomycosis-like pyoderma is a rare skin disorder most commonly caused by bacterial infection. It is usually diagnosed in immunocompromised patients. We report a case of BLP in an immunocompetent woman, who presented with a 6-week history of verrucous cutaneous plaque of the left wrist.
\end{abstract}

\section{Title: Blastomycosis-like pyoderma in an immunocompetent patient}

Authors: Sahli F, Litaiem N, Gara S, Charfi O, Rammeh S, Zeglaoui F

Author names and affiliations:

Fatma SAHLI ${ }^{1,2}$, MD, Noureddine LITAIEM $^{1,2}$, Soumaya GARA ${ }^{1,2}$,MD, Olfa CHARFI ${ }^{1,2}$,MD, Soumaya RAMMEH $^{2,3}, \mathrm{MD}$, Faten ZEGLAOUI ${ }^{1,2}, \mathrm{MD}$,

1. Department of Dermatology, Charles Nicolle Hospital, Tunis, Tunisia

2. Faculty of Medicine of Tunis, University of Tunis El Manar, Tunis, Tunisia

3. Department of Pathology, Charles Nicolle Hospital, Tunis, Tunisia

Corresponding author:

Fatma Sahli

Department of dermatology, Charles Nicolle Hospital, Tunis, Tunisia

Email address : sahlifatma@hotmail.com

Conflict of Interest Disclosure: None Declared

Funding Sources: None.

Keywords: dermatology, health maintenance, infectious diseases

Key Clinical Message: BLP is a rare and uncommon inflammatory disease of the skin.

The diagnosis is sometimes confusing and the clinic may suggest a pyoderma gangrenosum, mycobacterial infections or cutaneous tuberculosis.

\section{INTRODUCTION}

Blastomycosis-like pyoderma (BLP) is a rare cutaneous disorder. It presents as large verrucous plaques with multiple pustules and an elevated border [11References :Hongal AA, Gejje S. Blastomycosis-Like PyodermaA Rare Case Report. J Clin Diagn Res. 2016 Oct; 10(10): WD03-WD04.]. It is considered to be an exaggerated inflammatory response to bacteria, especiallyStaphylococcus aureus . 
BLP often occurs in patients with impaired immunological capacity [22Guidry JA, Downing C, Tyring SK. Deep Fungal Infections, Blastomycosis-Like Pyoderma, and Granulomatous Sexually Transmitted Infections. Dermatol Clin. 2015;33(3):595-607.]. We describe a rare case of BLP in an immunocompetent female patient.

\section{CASE REPORT}

A 65-year-old woman with diabetes mellitus type 2 presented with a 6-week history of verrucous cutaneous plaque of the anterior aspect of the left wrist. The lesion was painful and ulcerated. The patient denied any history of trauma or contact with patients with tuberculosis. Dermatological examination revealed an irregular, erythematous, and vegetating plaque of the right wrist, measuring $10 \times 4 \mathrm{~cm}$, with elevated and erythematous borders with multiple pustules of different sizes and central ulceration (figure 1 ). There was no palpable lymphadenopathy and physical examination was otherwise unremarkable.

Bacterial culture from a pus swab identified Staphylococcus aureus. Histopathological examination of a skin biopsy specimen showed acanthosis of the epidermis with inflammatory infiltration of the dermis made of lymphocytes, neutrophils, plasma cells, and histiocytes. The search of Leishmania by direct microscopy and polymerase chain reaction was negative.

The diagnosis of BLP was made. The patient received amoxicillin (3gr daily) with clavulanic acid (62.5 mg daily) and local wound care for 20 days. Substantial improvement was seen after 6 days (figure 2 ). A total resolution was obtained and no recurrence was noted after 6 months (figure 3 ).

\section{DISCUSSION}

BLP is an exaggerated chronic inflammatory reaction due to a bacterial infection [1]. It presents as vegetating skin lesions similar to blastomycosis or warty tuberculosis [2]. BLP is usually diagnosed in immunocompromised patients [2]. In these cases, BLP is related to several factors: HIV infection, alcoholism, neoplasia, malnutrition, and immunosuppressant drugs [2]. Cases of BLP in immunocompetent patients are rare. Local factors may be involved including injury, foreign bodies, tattoos, radiotherapy, and trauma [11Cecchi R, Bartoli L, Brunetti L, and Pavesi M. Blastomycosis-like pyoderma in association with recurrent vesicular hand eczema: good response to acitretin. Dermatol Online J . 2011 Mar; 17(3):9.Figure legend:Figure 1: Vegetating plaque of the right wrist with elevated borders, multiple pustules and central ulceration.Figure 2: Substantial improvement after 6-day antibiotic treatmentFigure 3: A resolution was noted after 6 months leaving a dyspigmented scar].

Numerous micro-organisms are associated with BLP includingStaphylococcus, Streptococcus pyogenes, Pseudomonas aeruginosa, Escherichia coli, and Candida albicans. However, Staphylococcus aureus is the most common causative micro-organism. The mechanism by which these microorganisms induce PLP remains unknown. Many believe that the microbiological agent is not directly responsible for the disease but by creating an immune dysfunction [3].

$\mathrm{Su}$ et al introduced [2] diagnostic criteria for BLP: (i) large verrucous plaques with multiple pustules and elevated border, (ii) histological evidence of pseudoepitheliomatous hyperplasia with abscesses, (iii) identifications of bacteria by tissue culture, (iv)negative culture for fungi and mycobacteria, (v) negative fungal serology tests and (vi) normal bromide and iodide blood levels. Our patient met four of these criteria.

The most effective treatment strategy includes targeted oral antibiotic therapy. Other treatment options could be considered for selected patients and include curettage, topical antibiotics, oral acitretin, intralesional or systemic corticosteroids, and carbon dioxide laser debridement [3].

Author contribution statement: Noureddine Litaiem wrote the first draft of the manuscript. Soumaya Gara, Olfa Charfi and Soumaya Rammeh managed the literature searches and analyses. Mariem Jones and Faten Zeglaoui revised the manuscript. All the authors contributed to and have approved the final manuscript. 

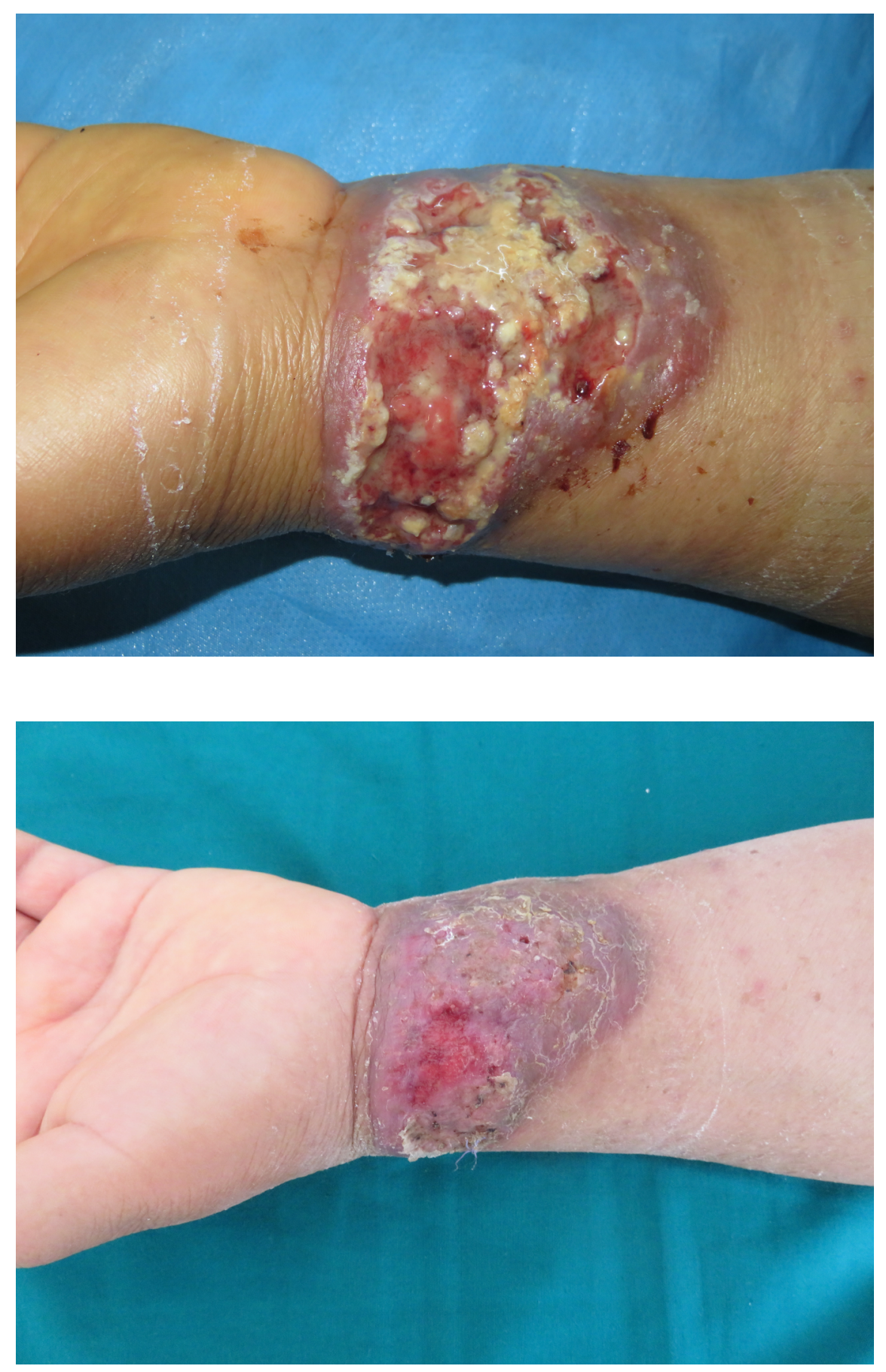


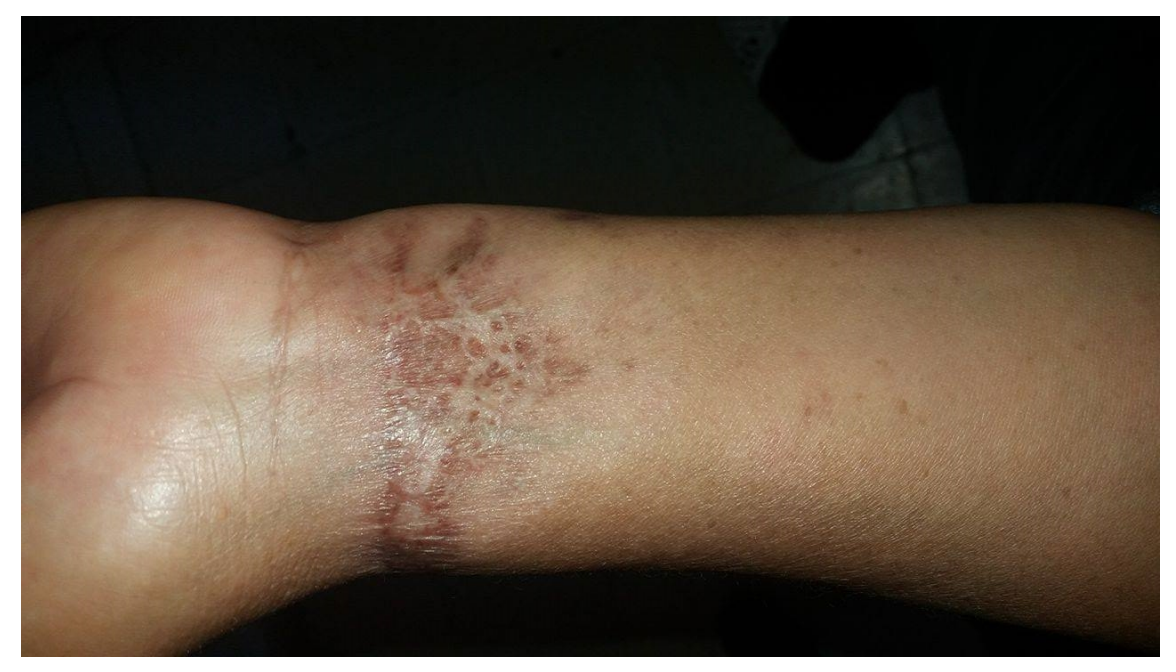

